(C) 2011 IEEE. Personal use of this material is permitted.

Permission from IEEE must be obtained for all other uses, in any current or future media, including reprinting/republishing this material for advertising or promotional purposes, creating new collective works, for resale or redistribution to servers or lists, or reuse of any copyrighted component of this work in other works. 


\title{
Feature Extraction of Non-Uniform Food Products Using RGB and RGB-D Data Combined With Shape Models
}

\author{
Helge A Wurdemann, Vahid Aminzadeh, Lei Cui, and Jian S Dai, IEEE Member.
}

\begin{abstract}
This research paper investigates a new 3D handling process in food industry: bin picking. Machines only function effectively when the input of product is physically well organised, well structured, and consistent. At many stages in a typical production line, foodstuffs are physically arranged as they move through a machine or equipment, however, this order is then lost again as products are ejected onto conveyors, bulked together into bins for transport, taken off-line for chilled storage. Bin picking is generally not solved for manufacturing parts. Unlike food ordering processes such as pick and place operations, vibratory feeders etc., this food handling operation has not been applied to food industry neither. A new approach is presented using the Microsoft Kinect ${ }^{\mathrm{TM}}$ sensor and Active Shape Models. By combining the new device that obtains an RGB and RGB-D image and the flexible shape model, it is possible to identify non-uniform food products that have a high variation in shape. The methodology of this system is presented. The experiments show the achievability of this new system.
\end{abstract}

\section{INTRODUCTION}

The Food Standards Agency of the United Kingdom defines the food industry as "that from farming and food production, packaging and distribution, to retail and catering"1 It uses large quantities of low-skilled labour, at relatively low unit cost compared to other sectors. In the medium term (5-7 years) the number of people willing to work for such low wages will decline and the industry will have to change to survive. Robotics and automation could provide an alternative to many labour intensive tasks; however, widespread uptake is inhibited by a combination of technical, financial and cultural problems [1] [2] [3]. Despite the potential benefits, the food industry uses only one robot per 1100 employees compared with one robot per 10 employees in the motor industry [4]. However, robotic systems have started to make inroads into applications in the food industry and it is expected that this sector will expand greatly [5].

Specialised machines and automation are commonly used for particular tasks within every industrial sector. These machines only function effectively when the input of product is physically well organised, well structured, and consistent. At many stages in a typical production line, foodstuffs are physically arranged as they move through a machine

This work was supported by the Engineering and Physical Sciences Research Council (EPSRC) of the United Kingdom under grant number EP/F031394/1

Helge A Wurdemann, Vahid Aminzadeh, and Prof. Jian S Dai are with the School of Natural and Mathematical Science, Centre for Robotics Research, King's College London, University of London, Strand, London WC2R 2LS, United Kingdom helge. wuerdemannekcl.ac.uk

Dr. Lei Cui is with the Carnegie Mellon University, Robotics Institute, 5000 Forbes Avenue, Pittsburgh, PA 15213, USA

${ }^{1}$ Food Standards Agency: www.food.gov.uk, July 2009 or equipment, however, this order is then lost again as products are ejected onto conveyors, bulked together into bins for transport, taken off-line for chilled storage, etc. If automation technology is to accommodate the real world degrees of physical variation in input foodstuffs and supply arrangement, then generic tools and techniques need to be developed to describe the physical transition from process disarray to order. The problem is generic, but solutions are non-trivial because, firstly, food products can vary greatly from each other, even within the same food type. Secondly, food products are difficult to handle and susceptible to damage. Foodstuffs typically exhibit non-rigid, visco-elastic behaviours [6] that are temperature-dependent [7]. Thirdly, mechanical properties of the handled components are temperature dependent as well, and, finally, equipment design must be hygienic and resistant to the rigours of a food production environment.

Solutions such as pick and place operations [8], multihead weighers, vibratory bowl feeder, etc. have already been applied to food industry [9]. Bin picking is a robot based singulation of objects that are randomly distributed in a three dimensional, limited space that has not yet been solved successfully [10]. Vision algorithms recognise pre-defined points of items and pick each. During this process, the robot that is provided with an individual end-effector should avoid any collision with remaining the items in the bin. Since the location of the objects is always different from the previous one, bin picking is based on an image processing system routinely analyses features of the parts to determine their orientation and location. Once the orientation of a part is determined, the part often requires reorientation for further processing.

\section{BACKGROUND}

Work has been reported since the early 1970s [11] [12] [13]. Success has been limited due to the inherent difficulties of the processing speed of the collected data, inability to isolate perspective and illumination effects. In [14], passive vision under the guide of analysis of simple optical flow is introduced. Optical flow is estimated by computing the time derivative of a sequence of images by matching between contours in images that have been generated from the zero crossings of Laplacian of Gaussian-filtered images. The technique can be improved by other visual cues. The evaluation of industrial parts using the Extended Gaussian Image is also the main focus in [15]. A segmentation that is applied to robotic 3D handling using vacuum grippers is able to detect edges in [16]. Furthermore, a region growing 
algorithm obtains the final segmented image. In doing so, a gripping point is determined for the vacuum end-effector. This algorithm is only tested on objects with obvious edges and plain shapes. In [17], another vision system is tested on non-complex manufacturing parts like multiple cylinders. A combination of range and intensity images is presented. The range image processing seems to be efficient. The more complex the handled parts are the more difficult bin picking gets [18]. This is why stereo vision-based algorithms only concentrate on object features. Ohba and Ikeuchi [19] proposed a new method called "Eigen-window" which recognises partly occluded objects in a bin. This is achieved by saving multiple appearances of the object in the Eigenspace. Berger, Bachler, and Scherer [20] split the task up into three parts: Firstly, a surface is determined to grasp a random part. Then, it is removed from the container. The key step is a system that uses a geometry integrated eigenspace approach to estimate the pose of the object. Tests prove the algorithm for manufacturing rigid objects. A similar approach is shown in [21]. Here, the pose estimation works for objects stacked in a pile, with cluttered background and different scale. A new sensor, solid state camera, for bin picking is introduced in [22]. This system picks and places randomly oriented but known polyhedral objects. Hereby, a model based and model free algorithm is tested in order to try handling more general objects. The model based solution runs reliable. Since Harmonic Shape Contexts are invariant to scale, translation and 3D rotation, Kirkegaard and Moeslund use this idea to estimate the pose. Objects are found one by one which meets the definition of bin picking. Additional investigation has to be the focus of further research. In [23], an ellipse fitting algorithm is presented. The Kalman estimator determines the pose of the object at the moment of pick up considering the noise that is caused by the motion of the conveyor belt since the parts are located there. Thielemann et al. [24] also remove industrial components from a conveyor belt using structured light and 3D pose estimation via CAD modelling. This literature review shows the importance of vision algorithms as part of the bin picking task. Dealing with certain objects in the bin requires mostly a certain and individual type of solution. In [25], a CAD model is needed in order to extract the best matching pose of an object within a range image obtained by a range sensor. In contrast to this $2.5 \mathrm{D}$ vision system, a 3D laser measurement method is presented in [26]. This technique also uses the data of 3D CAD models. A calibration of a Micro Electro Mechanical System (MEMS) scanner grating projector is implemented for a shape measurement system using the whole-space tabulation method (WSTM) and tested on a binpicking system [27]. The effectiveness is demonstrated for pieces of Japanese chess. In 2009, "Assembly Automation" [28] published a special issue on this kind of process. This underlines the up-to-date interest.

This paper is to provide a bin picking approach and a leading edge methodology to underpin the 3D physical creation task. A flow chart illustrates the sequence of the food ordering process. Further, the Microsoft Kinect ${ }^{\mathrm{TM}}$ sensor is introduced. The new device provides the input data for the Active Shape Model (ASM) algorithm in order to extract features of non-uniform food products. By aligning the model to a captured image of the bin and adding the depth information from the IR sensor of the Kinect ${ }^{\mathrm{TM}}$, it is possible to obtain the grasping points for the robot to pick the food products. In section III, the pinhole camera model is used for the 2-dimensional RGB cameras calibration, whereas the depth sensor is calibrated by a quadratic equation. The image procession part introduces ASMs, that will detect features from the top layer of the bin. Experiments prove the achievement of this technique.

\section{METHODOLOGICAL COMBINATION OF DEPTH DATA AND ACTIVE SHAPE MODELS}

The sequence of the bin picking process is shown in Fig. 1. The system is separated into the capturing of the RGB and RGB-D data and image pre-procession on the one hand. On the other hand, the ASM algorithm extracts the features of food objects using a priori knowledge.

1) Part A: The Kinect ${ }^{\mathrm{TM}}$ takes an overhead RGB and RGB-D image of the bin. If the maximum distance of the depth image equals the minimum distance, the bin is empty and the program will discontinue. Otherwise the top layer of the RGB image is filtered using the RGB-D image. The preprocessed data is transferred to the ASM algorithm.

2) Part B: The ASM aligns the trained model to the top layer of the bin. It will return the location and orientation of the feature. Combined with the depth data from the IR sensor, it is possible to identify the grasping points for the end effector of the robot in use.

These two parts of the process will be explained in more detail in the next two sections.

\section{RGB AND RGB-D IMAGE DATA USING THE MICROSOFT KINECT TM}

The RGB and RGB-D capturing device was launched in the UK early November 2010. The vision devices, that are located on a horizontal line, are connected to a small base with a motorised tilt mechanism. The Kinect ${ }^{\mathrm{TM}}$ consists of an RGB camera, depth sensor and multi-array microphone (Fig. 2). This chapter describes the functionality and ability of the device as well as the calibration.

\section{A. Design and Functionality}

The RGB images obtained by the colour CMOS camera have 8 -bit resolution $(640 \times 480$ pixels $)$. An extracted RGB image can be seen in Fig. 3(a). The CMOS sensor that will receive the IR light from the transmitter provides input for the depth map with 11-bit resolution $(320 \times 240$ pixels). However in this paper an 8 -bit resolution $(640 \times 480$ pixels) will be extracted (Fig. 3(b)). The principle of the Kinect ${ }^{\mathrm{TM}}$ sensor is as follows: Between the IR transmitter, sending out structured light, and receiver is a small angle. Also, the IR sensor should be provided with a band-pass filter in order to capture the IR light only. Using triangulation the depth can be recalculated. 


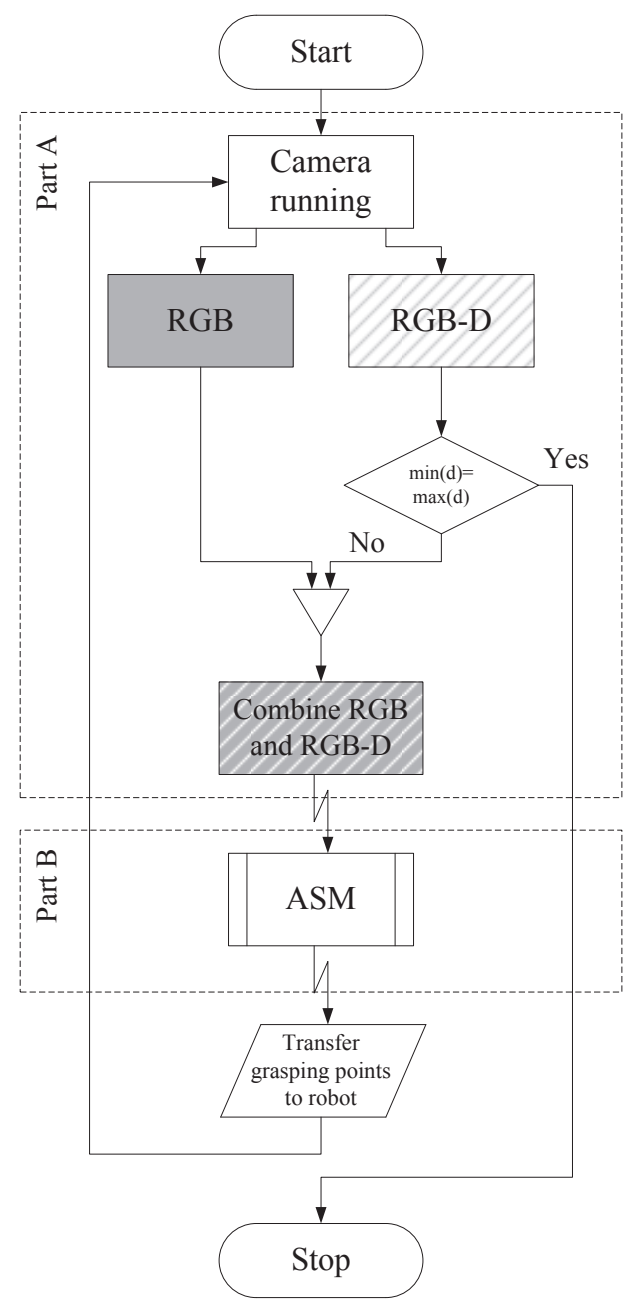

Fig. 1. Sequence of Bin Picking Operation Analysing RGB and RGB-D Using the Active Shape Model

\section{B. CMOS Camera Calibration}

The two CMOS cameras are calibrated using the widely known pinhole camera model [29]. Regarding the extrinsic parameters, the RGB camera will be used as the world coordinate frame, so that the depth sensor needs to be translated by $25 \mathrm{~mm}$ in horizontal direction. The intrinsic matrix $\mathbf{M}_{\text {Intrinsic }}$ is described by the focal length $f_{x}$ and $f_{y}$ and the principle point $p_{x}$ and $p_{y}$, so that everything adds up to the following camera matrix:

$$
\mathbf{M}_{\text {Intrinsic }}=\left[\begin{array}{ccc}
f_{x} & 0 & p_{x} \\
0 & f_{y} & p_{y} \\
0 & 0 & 1
\end{array}\right]
$$

In order to consider non-linear effects, the intrinsic matrix has to be multiplied with the radial distortion vector $\mathbf{r}_{\mathrm{d}}$ :

$\mathbf{r}_{\mathrm{d}}=\left[\begin{array}{c}d_{x}\left(x^{2}+y^{2}\right)^{3}+d_{y}\left(x^{2}+y^{2}\right)^{2}+d_{z}\left(x^{2}+y^{2}\right)+1 \\ d_{x}\left(x^{2}+y^{2}\right)^{3}+d_{y}\left(x^{2}+y^{2}\right)^{2}+d_{z}\left(x^{2}+y^{2}\right)+1 \\ 1\end{array}\right]$, where

$$
\left[\begin{array}{l}
x \\
y
\end{array}\right]=\left[\begin{array}{l}
X / Z \\
Y / Z
\end{array}\right]
$$

and where $X, Y, Z$ is a point in the camera reference frame. For the RGB camera, the intrinsic parameters are:

$$
\begin{gathered}
\mathbf{M}_{\text {Intrinsic, } R G B}=\left[\begin{array}{ccc}
522.82 & 0 & 320.61 \\
0 & 521.63 & 242.00 \\
0 & 0 & 1
\end{array}\right], \\
\mathbf{d}_{\mathrm{RGB}}=\left[\begin{array}{c}
1.12 \\
-0.91 \\
0.26
\end{array}\right],
\end{gathered}
$$

and for the IR sensor:

$$
\begin{gathered}
\mathbf{M}_{\text {Intrinsic,RGB-D }}=\left[\begin{array}{ccc}
557.14 & 0 & 304.44 \\
0 & 556.75 & 229.18 \\
0 & 0 & 1
\end{array}\right], \\
\mathbf{d}_{\mathrm{RGB}-\mathrm{D}}=\left[\begin{array}{c}
-0.2 \\
0.54 \\
-0.48
\end{array}\right] .
\end{gathered}
$$

\section{Mapping Distance versus Hue}

The depth image shows a certain RGB color sequence going from close to deep. As the distance between an object and the sensor increases, the order is as follows: magenta $(1,0,1)$, blue $(0,0,1)$, cyan $(0,1,1)$, green $(0,1,0)$, yellow $(1,1,0)$, red $(1,0,0)$, where $R, G, B \in[0,1]$. This can be written in cylindrical-coordinate representations by calculating the hue, saturation and lightness value in the HSV colour space. The three equations are given by [30]:

$$
\begin{aligned}
& H=\left\{\begin{array}{cc}
0^{\circ} & \ldots \\
60^{\circ}\left(0+\frac{G-B}{\max (R, G, B)-\min (R, G, B)}\right) & \ldots \\
60^{\circ}\left(0+\frac{B-R}{\max (R, G, B)-\min (R, G, B)}\right) & \ldots \\
60^{\circ}\left(0+\frac{R-G}{\max (R, G, B)-\min (R, G, B)}\right) & \ldots
\end{array}\right. \\
& \ldots \quad \text {, if } R=G=B \\
& \ldots \quad \text {, if } \max (R, G, B)=R \\
& \ldots \quad \text {, if } \max (R, G, B)=G \\
& \ldots \quad \text {, if } \max (R, G, B)=B
\end{aligned}
$$

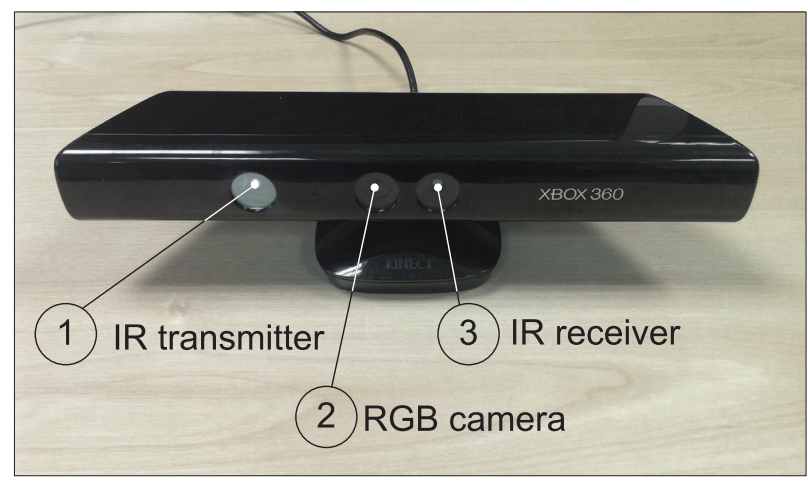

Fig. 2. Sensor Arrangement of the Microsoft Kinect ${ }^{\mathrm{TM}}$ 


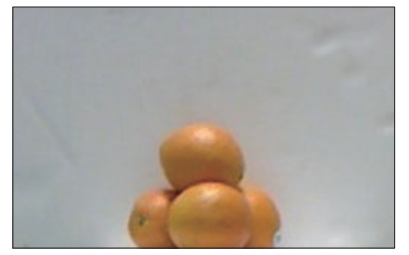

(a) RGB image

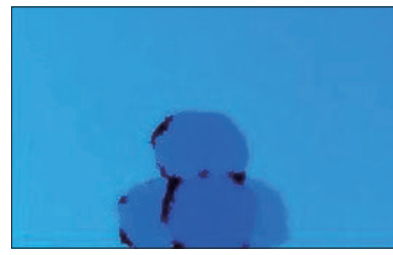

(b) RGB-D image
Fig. 3. RGB and Depth Image Capture

$$
\begin{array}{cl}
S=\left\{\begin{array}{cl}
0^{\circ} & , \text { if } R=G=B \\
\frac{\max (R, G, B)-\min (R, G, B)}{\max (R, G, B)} & , \text { otherwise } \\
V=\max (R, G, B)
\end{array}\right.
\end{array}
$$

Fig. 4 plots the distance $d=[70,170]$ in $[\mathrm{cm}]$ against the

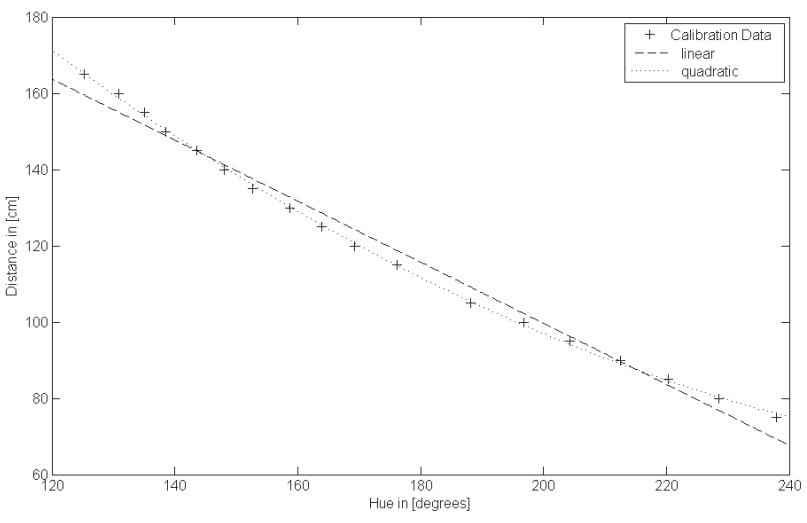

Fig. 4. Linear and Quadratic Interpolation between $d$ and $H$

hue value $H$ in $\left[^{\circ}\right.$. Unlike a linear approximation, a quadratic equation describes the ratio between the distance and the hue value more accurately:

$$
d=0.0032 \cdot H^{2}-1.9492 \cdot H+359.26
$$

Equation 11 allows the computation of distance based on quadratic relationship to hue.

\section{SHAPE-BASED MODEL FOR FEATURE EXTRACTION}

In the field of image processing there are well-known methods for manual, semi-automatic, and fully-automatic segmentations. They can generally be divided into pixel, edge, and regionbased methods. In addition, it is distinguished between model-based techniques, that integrate a priori knowledge about the segmented area, and texturebased techniques, that consider the inner texture of the area. Since this bin-picking research deals with food products that have a variation in shape and texture, a model-based image processing algorithm seems to be ideal to solve these difficulties and identify products in the bin. The ASM by Cootes et al. [31] is introduced here which is commonly used in medical technology and face recognition. More details can be found in [32].

\section{A. Training Shapes for Features of Non-Uniform Products}

In order to localise food products in images, models need to be extracted from training images. These models contain training sets of different shapes of the processed food product. A finite number $n$ of landmarks are placed on the boundary of the object, to describe its shape. The $x$ - and $y$ coordinates of a certain shape are saved in a two-dimensional vector $\mathbf{x}_{i}$ :

$$
\mathbf{x}_{i}=\left(x_{1}, \ldots, x_{n}, y_{1}, \ldots y_{n}\right)^{T}
$$

After applying the Principle Component Analysis (PCA) to the $i=[1, N]$ training images, the following will result as a mathematical transformation:

$$
\mathbf{x}=\overline{\mathbf{x}}+\mathbf{P}_{\mathrm{s}} \mathbf{b}_{\mathrm{s}}
$$

By varying the shape parameters of vector $\mathbf{b}_{\mathrm{s}}$, the shape $\mathbf{x}$ will vary. Defining the elements of $\mathbf{b}_{\mathrm{s}}$, the shapes are similar to the training images after the PCA.

\section{B. Feature Extraction Using A Priori Knowledge}

The model that is created by the PCA can be aligned to an unknown image, as long as this object exists within the image. Thereby, it is not only necessary to vary the shape parameter $\mathbf{b}_{\mathrm{s}}$, but also the scale, rotation and translation. This can be expressed by a transformation matrix:

$$
\mathbf{T}_{X_{t}, Y_{t}, s, \theta}\left(\begin{array}{l}
x \\
y
\end{array}\right)=\left(\begin{array}{l}
X_{t} \\
Y_{t}
\end{array}\right)+\left(\begin{array}{cc}
s \cos \theta & s \sin \theta \\
-s \sin \theta & s \cos \theta
\end{array}\right)\left(\begin{array}{l}
x \\
y
\end{array}\right)
$$

The transformation matrix 14 is multiplied by the created model 13. The new object coordinates are saved in $\mathbf{Y}$ and compared with the model until the model matches the new object. This happens as the minimum of the difference

$$
\left|\mathbf{Y}-\mathbf{T}_{X_{t}, Y_{t}, s, \theta}\left(\overline{\mathbf{x}}+\mathbf{P}_{\mathrm{s}} \mathbf{b}_{\mathrm{s}}\right)\right|^{2}
$$

is determined by iterations.

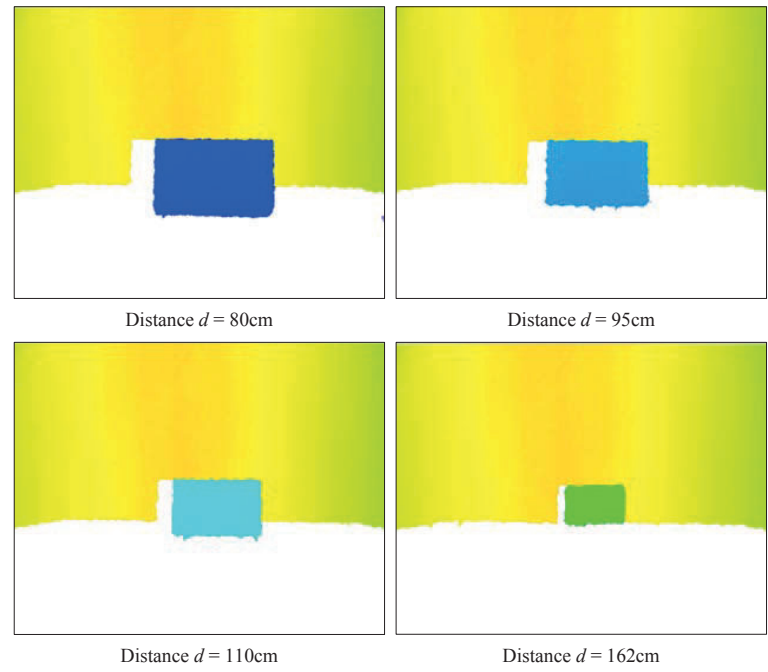

Fig. 5. Distance Calculation Using RGB to HSV Converted Images 


\section{EXPERIMENTS}

\section{A. HSV Distance Validation}

Since the IR depth sensor is calibrated for a distance between $70 \mathrm{~cm}$ and $170 \mathrm{~cm}$, tests are taken within this interval. Four samples can be seen in Fig. 5. The object in the centre of the image is located for a distance $d=$ $80,95,110,162 \mathrm{~cm}$ away from the Kinect ${ }^{\mathrm{TM}}$. Table I shows

TABLE I

RGB-D SAMple Distance Calculation

\begin{tabular}{c|c|c|c|c|c} 
Sample & $R$ & $G$ & $B$ & $H$ in $\left[^{\circ}\right]$ & $d$ in $[\mathrm{cm}]$ \\
\hline \hline 1 & 0.00 & 0.24 & 1.00 & 225.88 & 81.67 \\
\hline 2 & 0.01 & 0.64 & 1.00 & 202.06 & 95.60 \\
\hline 3 & 0.00 & 0.98 & 1.00 & 181.41 & 110.60 \\
\hline 4 & 0.00 & 1.00 & 0.12 & 127.29 & 162.81
\end{tabular}

step-by-step samples of the translation from the RGB color space to the HSV colour space into distance $d$. As mentioned before, the hue value is of special interest because this is related to the distance $d$ by 11 . Using this interpolation, the distance can be calculated. Compared to the measured distance $d$, the average error is $1.01 \%$. Implementing the calibrated quadratic distance equation for $d$, Equation 11, Table I and Fig. 5 present the expected distance valuation of the tracked obstacle.

\section{B. $3 D$ Reconstruction of Bin Volume}

From the 3D data gained from the RGB-D sensor, it is possible to generate an HSV image. The new image specifies a mesh by a 3-tuple: $x-, y$-coordinate and a normalised hue value. Fig. 6 shows the depth map of the top view of Fig. 3 . As it can been seen, the food products in different layers can be distinguished.

\section{Results of the New Bin Picking Approach}

In order to extract the top layer image of the RGB image, the RGB-D image (Fig. 7(a)) is converted into an HSV image (Fig. 7(b)) using 8-10. Since the hue value $H$ obtains the

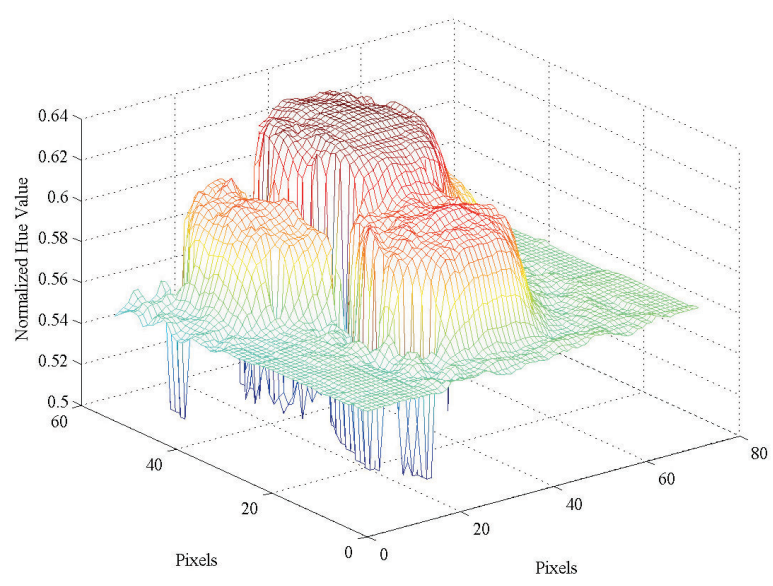

Fig. 6. 3D Mesh Generated by 3-tuple $(x, y, H)$

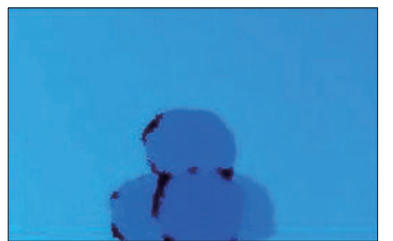

(a) RGB-D image

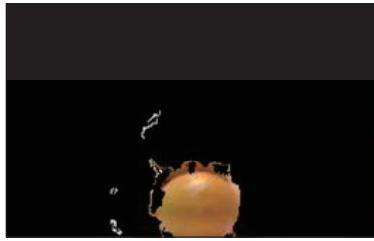

(c) Top Layer Reduction

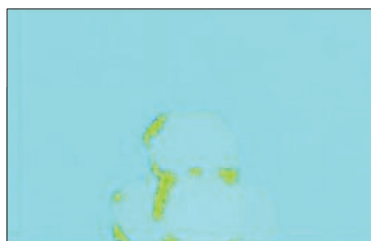

(b) HSV image

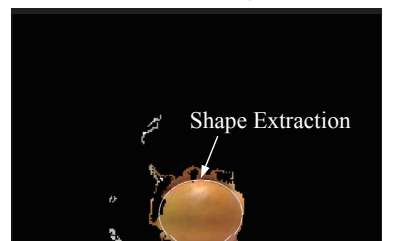

(d) ASM Feature Extraction
Fig. 7. Feature Extraction Sequence Results

distance via the quadratic interpolation 11, the top layer of the bin can be filtered as shown in Fig. 7(c). Now, the ASM that was generated by training images in advance is aligned to the new unseen image using 15 and the shape of the top level orange is extracted (Fig. 7(d)).

\section{CONCLUSIONS}

This paper presents a novel bin picking operation of nonuniform products for food industry. The technique uses the new device Microsoft Kinect ${ }^{\mathrm{TM}}$ in order to exploit the RGB and RGB-D images for the food ordering task. Before any image processing is possible the image inputs are calibrated to acquire the image in a pinhole model with intrinsic calibration. Using a HSV cylindrical-coordinate mapping space, a quadratic distance estimation model is presented to resolve the estimation of a food product distance from the sensor and filter the top layer of the bin. The ASM that is commonly used in medical technology and face recognition is introduced. This algorithm is trained with shapes of the non-uniform food product that needs to be extracted. The experimental results show one sequence of the process.

The images and results demonstrate the potential of this new bin picking methodology. It shows the feasibility of using a combination of the Microsoft Kinect ${ }^{\mathrm{TM}}$ sensor and the ASM for extracting features of non-uniform food products in order to create physical structure from 3D disarray.

\section{ACKNOWLEDGMENTS}

In particular we would like to thank the Engineering and Physical Sciences Research Council (EPSRC) of the United Kingdom under grant number EP/F031394/1 for the financial support of this project as well as Dr. Graham Purnell from the Grimsby Institute and John Reed from Silsoe Technology Ltd., the co-investigators of this joined research project.

\section{REFERENCES}

[1] A. Emerald, "Snubbing robotic innovation," Strategic Direction, vol. 22 , pp. 30-31, 2006.

[2] R. Butler, "What's cooking?," Professional Engineering, vol. 19, no. 6, pp. 44-46, 2006. 
[3] G. Purnell, "Robotic response to processing challenges," Food Processing Intelligence, pp. 37-39, 2005.

[4] J. Reed, "Robotics in the food industry," in Proceedings of Food and Drink Seminar, (Bologna), 2005.

[5] K. Young, "Home improvements," The British Automation and Robot Association, p. 53, 2007

[6] B. Evans and P. Brett, "Computer simulation of the deformation of dough-like materials in a parallel plate gripper," Journal of Engineering Manufacture, vol. 210, no. 2, pp. 119-126, 1996.

[7] T. Brown, S. James, and G. Purnell, "Cutting forces in foods: Experimental measurements," Journal of Food Engineering, vol. 70, no. 2, pp. 165-170, 2005.

[8] A. Aminzadeh, H. Wurdemann, J. Dai, J. Reed, and G. Purnell, "A new algorithm for pick-and-place operation," Industrial Robot: An International Journal, vol. 37, no. 6, p. 527531, 2010.

[9] H. Wurdemann, A. Aminzadeh, J. Dai, J. Reed, and G. Purnell, "Category-based food ordering processes," Trends in Food Science \& Technology, vol. 22, pp. 14-20, 2011.

[10] H. Wurdemann, A. Aminzadeh, J. Dai, J. Reed, and G. Purnell, "Introducing a new 3d ordering process for discrete food products using food categorisation," Industrial Robot: An International Journal, vol. 37, no. 6, p. 562570, 2010.

[11] J. Poirier, "Robot vision and the bin of parts problem," Master's thesis, Kingston, RI: Dept. of Electrical Engg., Univ. of Rhode Island, 1985.

[12] J. Birk, R. Kelley, and L. Wilson, "Acquiring workpieces: Three approaches to using vision," in International Symposium on Industrial Robots, 1978.

[13] R. Kelley, J. Birk, H. Martins, and D. Duncan, "A robot system which feeds workpieces directly from bins to machines," in International Symposium on Industrial Robots, 1978.

[14] D. Vernon and M. Tistarelli, "Using camera motion to estimate range for robotic parts manipulation," IEEE Transaction on Robotics and Automation, vol. 6, pp. 509-521, 1990.

[15] A. Distante, N. Ancona, G. Attolico, L. Caponetti, M. Chiaradia, and E. Stella, "A model-based 3-d vision system for bin-picking," IEEE Transaction on Circuits and Systems, vol. 35, pp. 545-553, 1988.

[16] E. Al-Hujazi and A. Sood, "Range image segmentation with applications to robot bin-picking using vacuum gripper," IEEE Transaction on Systems, Man, and Cybernetics, vol. 20, pp. 1313-1325, 1990.

[17] K. Umeda and T. Arai, "Industrial vision system by fusing range image and intensity image," in IEEE International Conference on Multisensor Fusion and Integration for Intelligent Systems, 1994.

[18] K. Rahardja and A. Kosaka, "Vision-based bin-picking: Recognition and localization of multiple complex objects using simple visua cues," in IEEE/RSJ International Conference on Intelligent Robots ans Systems, 1996

[19] K. Ohba and K. Ikeuchi, "Recognition of the multi specularity objects for bin-picking task," in IEEE/RSJ International Conference on Intelligent Robots and Systems, 1996.

[20] M. Berger, G. Bachler, and S. Scherer, Intelligent Problem Solving. Methodologies and Approaches, ch. Vision Guided Bin Picking and Mounting in a Flexible Assembly Cell, pp. 109-117. Springer-Verlag Berlin Heidelberg, 2000.

[21] R. Soderberg, K. Nordberg, and G. Granlund, IbPRIA 2005, ch. An Invariant and Compact Representation for Unrestricted Pose Estimation, pp. 3-10. 2005 .

[22] S. Kristensen, S. Estable, M. Kossow, and R. Brosel, "Bin-picking with a solid state range camera," Robotics and Autonomous Systems, vol. 35, pp. 143-151, 2001.

[23] S. Noh, S. Park, Y. Han, and H. Hahn, "Pose estimation of top object on stack of elliptical objects based on ellipse fitting and kalman estimator," in 6th WSEAS International Conference on Signal processing, Robotics and Automation, 2007.

[24] J. Thielemann, O. Stkotheim, J. O. Nygaard, and T. Vollset, "System for conveyor belt part picking using structured light and $3 \mathrm{~d}$ pose estimation," in Proceedings of SPIE, the International Society for Optical Engineering, 2009.

[25] K. Boehnke, "Object localization in range data for robotic bin picking," in Proceedings of the 3rd Annual IEEE Conference on Automation and Engineering, pp. 572-577, 2007.

[26] O. Kazuya, H. Toshihiro, F. Masakazu, S. Nobuhiro, and S. Mitsuharu, "Development for industrial robotics applications," IHI Engineering Review, vol. 42, no. 2, pp. 103-107, 2009.

[27] D. Asai, T. Miyagi, M. Fujigaki, and Y. Morimoto, "Application to binpicking of shape measurement using whole-space tabulation method with mems scanner grating projector," Journal of JSEM, vol. 10 , no. Special Issue, pp. 186-191, 2010.

[28] A. Automation, Bin Picking and Flexible Gripping, vol. 29. 2009.

[29] J. Heikkil and O. Silven, "A four-step camera calibration procedure with implicit image correction," in Proc. IEEE Conference on Com puter Vision and Pattern Recognition, vol. 1, pp. 1106-1112, 1997.

[30] J. Park, G. N. DeSouza, and A. C. Kak, "Dual-beam structured-light scanning for 3-d object modeling," in Third International Conference on 3D Digital Imaging and Modeling, 2001.

[31] T. Cootes, G. Edwards, and C. Taylor, "Active appearance," Lecture Notes in Computer Science, vol. 1407, p. 484, 1998

[32] M. Stegmann, "Active appearance models: Theory, extensions and cases," Master's thesis, Informatics and Mathematical Modelling, Technical University of Denmark, 2004 